\title{
On Time Delay Estimation using an FIR Filter
}

\author{
H. C. So \\ Department of Electronic Engineering, City University of Hong Kong \\ Tat Chee Avenue, Kowloon, Hong Kong \\ Tel : (852) $27887780 \quad$ Fax : (852) $27887791 \quad$ email: hcso@ee.cityu.edu.hk
}

June 3, 2012

Keywords : Least Squares Filtering, Time Delay Estimation, Bias Removal

Abstract : A popular technique for time delay estimation is to use an FIR filter to model the time difference and the filter weights are interpolated with a sinc function to obtain the delay estimate. However, the sinc interpolator requires a sufficiently long filter length for accurate delay estimation. In this paper, we propose to process the filter weights via a least squares based method in order to acquire optimum delay estimates even with short filter lengths.

\section{Introduction}

The problem of estimating the differential delay between two noisy versions of the same signal received at two spatially separated sensors has attracted much attention in the literature [1]. Application examples include transmitter linearization [2], synchronization in communication systems [3], speech enhancement, determination of the centre of earthquakes, and source localization in sonar and radio systems [4].

Given the discrete-time outputs of the two sensors,

$$
r_{1}(k)=s(k)+n_{1}(k)
$$

and

$$
r_{2}(k)=\alpha s(k-D)+n_{2}(k), \quad k=0,1, \cdots, N-1
$$

where $s(k)$ is the real stationary random source signal while $n_{1}(k)$ and $n_{2}(k)$ are uncorrelated white Gaussian noises which are independent of $s(k)$. The $\alpha$ is the attenuation factor between the sensors and $N$ represents the number of samples collected at each sensor. Without loss of generality, we assume that the sampling period is unity second and $s(k)$ is bandlimited between $0 \mathrm{~Hz}$ and $0.5 \mathrm{~Hz}$. The aim is to find the time difference of arrival, $D$, from the received signals $r_{1}(k)$ and $r_{2}(k)$.

Generalized cross-correlation (GCC) [1] is one of the conventional methods for finding the time differences but it requires a priori statistics of the received signals in order to obtain 
accurate delay estimates. On the other hand, Chan et al introduced a parameter estimation approach [5] to approximate the time shift as an FIR filter, $W(z)=\sum_{i=-P}^{P} w_{i} z^{-i}$, in one of the receiver channel. Comparing with the GCC, this technique has the advantages of avoiding the need for spectral estimation from finite length data and capability of tracking nonstationary delays by making the filter adaptive [6]. Once the filter coefficients are determined, the delay estimate is obtained indirectly from $\left\{w_{i}\right\}$ by sinc interpolation [5]-[6]:

$$
\hat{D}=\arg \max _{t}\left\{\sum_{i=-P}^{P} w_{i} \operatorname{sinc}(t-i)\right\}
$$

where $\operatorname{sinc}(v)=\sin (\pi v) /(\pi v)$. However, it has been shown [5] that this interpolator is biased for finite $P$ and the delay bias decreases as the filter length increases. In this paper, a least squares based technique is proposed to process the filter weights in order to provide optimum delay estimation performance even if short filter lengths are employed.

\section{Proposed Method}

In the parameter estimation approach, the output of the filter $W(z)$ is subtracted from the other received signal to generate an error function $e(k)$. Without loss of generality, $e(k)$ is expressed as

$$
e(k)=r_{2}(k)-\sum_{i=-P}^{P} w_{i} r_{1}(k-i)
$$

Using the interpolation formula [5], $s(k-D)$ can be represented as

$$
s(k-D)=\sum_{i=-\infty}^{\infty} s(k-i) h_{i}
$$

where $h_{i}=\operatorname{sinc}(i-D)$. With the use of (4), squaring both sides of (3) and taking expectation yields

$$
E\left\{e^{2}(k)\right\}=C+\sigma_{n}^{2} \sum_{i=-P}^{P} w_{i}^{2}+\sum_{i=-P}^{P} \sum_{j=-P}^{P}\left(\alpha h_{i}-w_{i}\right)\left(\alpha h_{j}-w_{j}\right) R_{s s}(i-j)
$$

where $C$ is a function independent of $\left\{w_{i}\right\}, \sigma_{n}^{2}$ is the power of $n_{1}(k)$ and $R_{s s}(v)$ denotes the auto-correlation of $s(k)$ with a time shift of $v$. Differentiate $E\left\{e^{2}(k)\right\}$ with respect to each $w_{i}$ and equate the resultant expressions to zero, we obtain

$$
\sigma_{n}^{2} w_{i}^{o}+\sum_{j=-P}^{P}\left(\alpha h_{j}-w_{j}^{o}\right) R_{s s}(i-j)=0, \quad i=-P,-P+1, \cdots, P
$$

The minimum mean square estimates $\left\{w_{i}^{o}\right\}$ can be solved exactly if $\sigma_{n}^{2}, \alpha$ and the signal auto-correlation function are all known. To simplify the analysis, we consider the cases when $s(k)$ is weakly correlated, that is, $R_{s s}(0)>>R_{s s}(i)$ for $i \neq 0$, so that the optimum filter weights can be approximated as

$$
w_{i}^{o} \approx \lambda h_{i}, \quad i=-P,-P+1, \cdots, P
$$

where $\lambda=\alpha R_{s s}(0) /\left(\sigma_{n}^{2}+R_{s s}(0)\right)$. It is seen that $w_{j}^{o}$ consists of two components, $\lambda$, which is a scalar depends only on the signal-to-noise ratio (SNR) and $\alpha$, and $h_{i}$, which is a function of 
$D$. Based on this observation, we propose to estimate $D$ by minimizing the sum of squared differences between $w_{i}^{o}$ and a scaled sinc function as follows,

$$
\hat{D}^{o}=\arg \min _{\hat{\lambda}, \hat{D}}\{J(\hat{\lambda}, \hat{D})\}
$$

where

$$
J(\hat{\lambda}, \hat{D})=\sum_{i=-P}^{P}\left(w_{i}^{o}-\hat{\lambda} \operatorname{sinc}(i-\hat{D})\right)^{2}
$$

is characterized by the estimates of $\lambda$ and $D$. Solving for $\hat{\lambda}$, the optimum delay estimate is computed from

$$
\hat{D}^{o}=\arg \min _{\hat{D}}\left\{\sum_{j=-P}^{P}\left(w_{j}^{o}-\frac{\sum_{i=-P}^{P} w_{i}^{o} \operatorname{sinc}(i-\hat{D})}{\sum_{i=-P}^{P} \operatorname{sinc}^{2}(i-\hat{D})} \operatorname{sinc}(j-\hat{D})\right)^{2}\right\}
$$

In practical applications, least squares estimates of $\left\{w_{i}\right\}$, instead of the minimum mean square estimates, are employed in (10) and they are calculated as

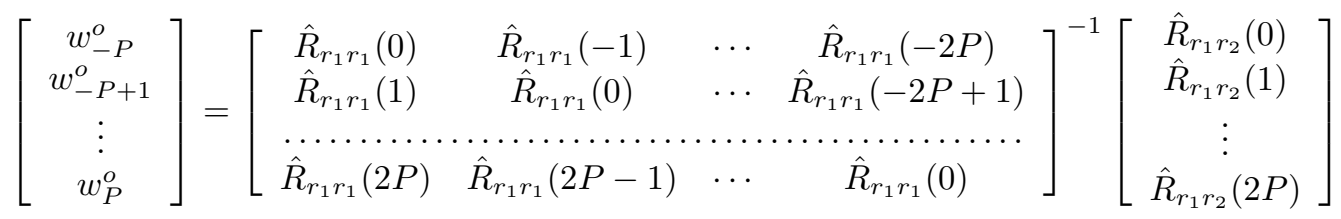

where $\hat{R}_{r_{1} r_{1}}(v)$ and $\hat{R}_{r_{1} r_{2}}(v)$ are the estimated auto-correlation function of $r_{1}(k)$ and crosscorrelation function of $r_{1}(k)$ and $r_{2}(k)$, respectively, from the $N$ measurements of $r_{1}(k)$ and $r_{2}(k)$.

\section{Performance Analysis}

The variance of $\hat{D}^{o}$ is expressed as $[6]$

$$
\operatorname{var}\left(\hat{D}^{o}\right)=\left.\frac{E\left\{\left(\frac{\partial J(\hat{\lambda}, \hat{D})}{\partial \hat{D}}\right)^{2}\right\}}{\left(E\left\{\frac{\partial^{2} J(\hat{\lambda}, \hat{D})}{\partial \hat{D}^{2}}\right\}\right)^{2}}\right|_{\hat{\lambda}=\lambda, \hat{D}=D}
$$

For ease of analysis, we assume that $s(k)$ is a white process with variance $\sigma_{s}^{2}, \alpha=1$ and the powers of $n_{1}(k)$ and $n_{2}(k)$ are identical. The numerator and denominator can be shown to be

$$
\left.E\left\{\left(\frac{\partial J(\hat{\lambda}, \hat{D})}{\partial \hat{D}}\right)^{2}\right\}\right|_{\hat{\lambda}=\lambda, \hat{D}=D}=4 \lambda^{2} \sum_{i=-P}^{P} \operatorname{var}\left(w_{i}^{o}\right) f^{2}(i-D)
$$

and

$$
\left.\left(E\left\{\frac{\partial^{2} J(\hat{\lambda}, \hat{D})}{\partial \hat{D}^{2}}\right\}\right)^{2}\right|_{\hat{\lambda}=\lambda, \hat{D}=D}=2 \lambda^{2} \sum_{i=-P}^{P} f^{2}(i-D)
$$


where $f(v)=(\cos (\pi v)-\operatorname{sinc}(v)) / v$ and $\operatorname{var}\left(w_{i}^{o}\right) \approx(1+2 \mathrm{SNR}) /\left(N(1+\mathrm{SNR})^{2}\right)$ represents the variance of $w_{i}^{o}, i=-P,-P+1, \cdots, P$, with SNR $=\sigma_{s}^{2} / \sigma_{n}^{2}$. Substituting (13) and (14) into (12) gives

$$
\operatorname{var}\left(\hat{D}^{o}\right) \approx \frac{(1+2 \mathrm{SNR})}{N_{\mathrm{SNR}}^{2} \sum_{j=-P}^{P} f^{2}(j-D)}
$$

Since $\sum_{j=-P}^{P} f^{2}(j-D)$ is close to its limiting value $\lim _{P \rightarrow \infty} \sum_{j=-P}^{P} f^{2}(j-D)=\pi^{2} / 3$ even for a small $P$, and the Cramér-Rao lower bound (CRLB) for time delay estimation using white signals is equal to $3(1+2 \mathrm{SNR}) /\left(\pi^{2} N \mathrm{SNR}^{2}\right)$ [1], the delay variance of proposed method will approach the CRLB for a wide range of filter lengths.

\section{Numerical Examples}

Simulation tests were carried out to evaluate the performance of the proposed delay estimator using least squares filter weights. Comparisons of mean square delay errors were also made with the sinc interpolator and the CRLB. The searching procedures of $(2)$ and (10) were performed by the bisection method. The source signal was Gaussian distributed with unity power and different SNRs were obtained by proper scaling of the random noise sequences. For simplicity, the additive noises were assigned to have identical power. The time difference $D$ was set to $0.7 \mathrm{~s}$, the data length $N$ was 1000 and $\alpha=1$ was selected. The mean square delay errors obtained were based on 1000 independent runs.

In the first test, the source signal was a white process. Figure 1 shows the delay variances of the proposed method and sinc interpolator using $P=3$ and $P=15$, as well the CRLB, for SNR $\in[-10 \mathrm{~dB}, 20 \mathrm{~dB}]$. It can be seen that when SNR $\geq-6 \mathrm{~dB}$, the proposed approach was superior to the sinc interpolation method using $P=3$ and $P=15$, for the whole range of SNRs and for SNR $\geq 5 \mathrm{~dB}$, respectively. Furthermore, the proposed method with $P=3$ and $P=15$ had similar performance and both met the CRLB for $-6 \mathrm{~dB} \leq \mathrm{SNR} \leq 10 \mathrm{~dB}$, although the latter slightly outperformed the former at very high SNRs. Since the delay estimation performance of (10) was almost independent of $P$ for a wide range of SNRs, a short filter length can be used in practice in order to reduce computation. On the other hand, the sinc interpolator was very sensitive to $P$ and sufficiently long filter lengths are required to acquire accurate delay estimates, particularly for high SNR conditions.

The previous experiment was repeated for an autoregressive (AR) source signal, viz., $s(k)=$ $a s(k-1)+w(k)$ where $w(k)$ was a white Gaussian process and the AR parameter was $a=0.5$. The signal auto-correlation function was given by $R_{s s}(i)=a^{|i|} R_{s s}(0)$, which implies that $s(k)$ was not weakly correlated, and the corresponding CRLB was computed using [1]. Figure 2 plots the results of this test. Again, all methods had a threshold SNR of $-6 \mathrm{~dB}$ and the proposed scheme with $P=3$ and $P=15$ attained the CRLB for $-6 \mathrm{~dB} \leq \mathrm{SNR} \leq$ $10 \mathrm{~dB}$, and surpassed the sinc interpolator, particularly for $P=3$. For $\mathrm{SNR} \geq 10 \mathrm{~dB}$, the suggested method using $P=15$ had little improvement over that of $P=3$ and was close to the CRLB. This means that the searching procedure of (10) can work well for correlated source signals as well. 


\section{Conclusions}

Conventional parameter estimation approach for time delay estimation employs an FIR filter to approximate the time difference where it is determined by interpolating the filter weights with a sinc function. In this paper, a least squares based technique is suggested to efficiently process the filter coefficients for optimum delay estimation. It is shown that the proposed method can attain the CRLB and outperform the sinc interpolator for a wide range of SNRs and different source signals, and even when the filter length is short.

\section{References}

[1] G.C.Carter, Coherence and Time Delay Estimation: An Applied Tutorial for Research, Development, Test and Evaluation Engineers, IEEE press, 1993

[2] A.R.Mansell and A.Bateman, "Practical implementation issues for adaptive predistortion transmitter linearization," IEE Colloq. Linear RF Amplifiers and Transmitters, pp.5/1-5/7, London, April 1994

[3] S.R.Dooley and A.K.Nandi, "Adaptive time delay and frequency esitmation for digital signal synchronization in CDMA systems," Conf. Rec. of the 32th Asilomar Conf. Signals, Systems \& Computers, vol.2, pp.1838-1842, 1998

[4] K.Itoh, S.Watanabe, T.Sato and Y.Hoshiko, "Radio source positioning using received time of arrival differences," Proc. IEEE Veh. Tech. Conf. vol.4, pp.2063-2066, Sept. 1999

[5] Y.T.Chan, J.M.F.Riley and J.B.Plant, "Modeling of time-delay and its application to estimation of nonstationary delays," IEEE Trans. Acoust., Speech, Signal Processing, vol.29, no.3, pp.577-581, 1981

[6] F.A. Reed, P.L. Feintuch, N.J. Bershad, "Time delay estimation using the LMS adaptive filter - static behaviour," IEEE Trans. Acoust., Speech, Signal Processing, vol.29, no.3, 1981, pp.561-571 




Figure 1: Mean square delay errors versus SNR for white signal

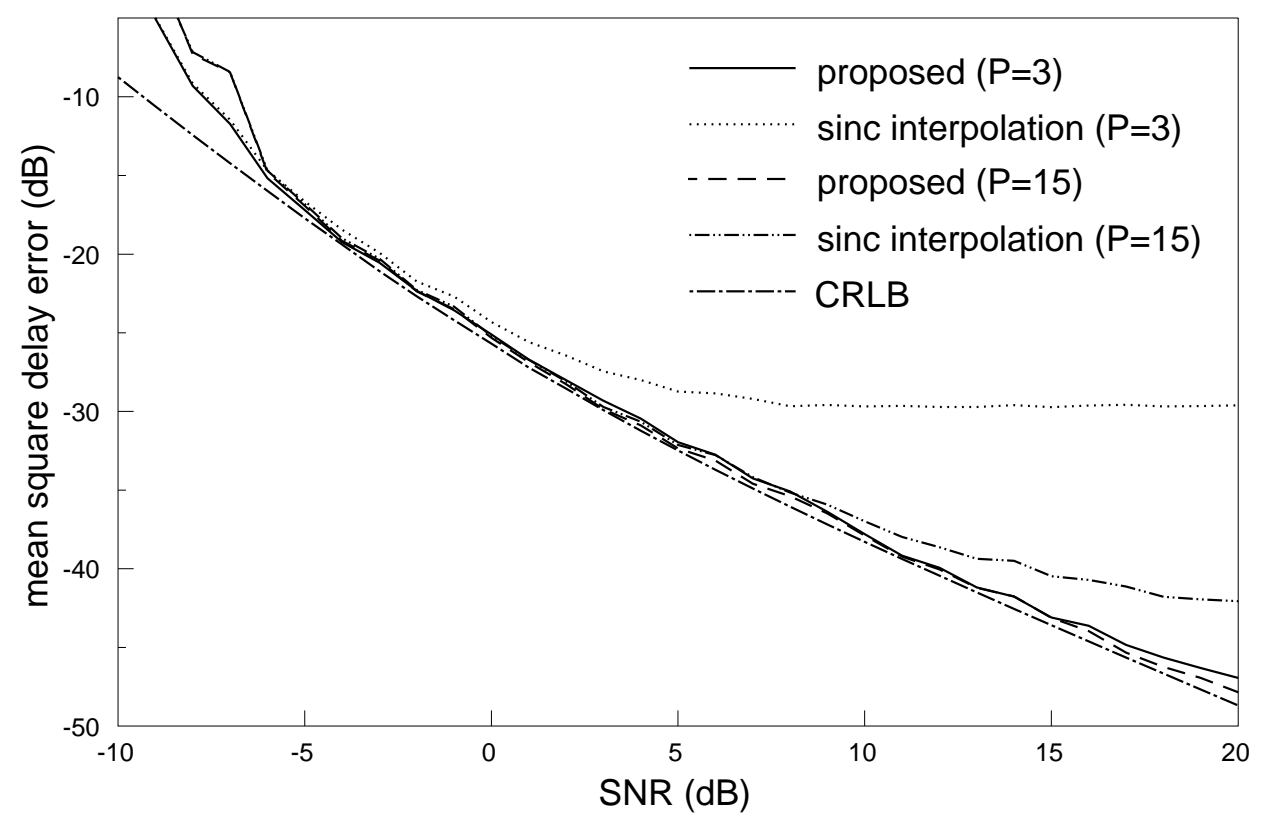

Figure 2: Mean square delay errors versus SNR for AR signal 